\title{
Linx
}

Revue des linguistes de l'université Paris X Nanterre

49 | 2003

L'actualité des notions d'interlangue et d'interaction exolingue

\section{Deux repères de recherche sur l'interaction exolingue}

Rémy Porquier

\section{CpenEdition}

Journals

Édition électronique

URL : http://journals.openedition.org/linx/538

DOI : $10.4000 / \operatorname{lin} \times .538$

ISSN : 2118-9692

Éditeur

Presses universitaires de Paris Nanterre

Édition imprimée

Date de publication : 1 décembre 2003

Pagination : 51-62

ISSN : 0246-8743

Référence électronique

Rémy Porquier, «Deux repères de recherche sur l'interaction exolingue », Linx [En ligne], 49 | 2003, mis en ligne le 08 mars 2011, consulté le 02 mai 2019. URL : http://journals.openedition.org/linx/538

DOI : $10.4000 / \operatorname{lin} x .538$ 


\title{
Deux repères de recherche sur l'interaction exolingue
}

\author{
Rémy Porquier \\ Université Paris X - UMR 7114
}

Cet article vise à signaler et à relier deux repères, l'un ancien, l'autre plus récent, dans la recherche sur l'acquisition des langues, les interlangues et l'interaction exolingue ${ }^{1}$. Maintes publications (Pujol et Véronique 1991, Ellis 1994, Véronique 1992 et 1997, Vogel, 1995, Selinker 1995, Gaonac'h et Perdue 2001, Rosen 2001, parmi bien d'autres) ont présenté sous divers angles, globalement ou partiellement, l'historique, l'itinéraire et des états et bilans des recherches menées en ce domaine entre la fin des années 1960 et la fin des années 1990.

Le propos est ici de leur apporter un éclairage complémentaire et une mise en perspective, d'une part à travers l'évocation d'une filiation de recherche et d'enquête de terrain remontant à l'antérieur des années 1970, d'autre part en évoquant des ouvertures relativement récentes (années 1990 et au-delà) sur les interactions exolingues en contexte professionnel. C'est-à-dire de part et d'autre de la riche période de recherche évoquée ci-dessus. On commentera brièvement en conclusion le lien, la complémentarité et la continuité entre ces deux repères de l'interaction exolingue au travail.

Si l'on accepte de caractériser la communication exolingue par une asymétrie entre les répertoires ou les compétences des participants, celle-ci peut concerner la langue ou les langues (langue non maternelle de l'un au moins des participants) de l'échange ou bien le degré d'expertise (spécialiste/non spécialiste, médecin/patient, etc.) dans le domaine de l'échange, ou les deux. Dans ce dernier cas, l'asymétrie peut être double, et croisée.

Les deux extraits de corpus ci-dessous fournissent une illustration préalable aux types d'interaction évoqués ensuite. L'extrait A provient du vaste corpus du programme de recherche ESF sur l'acquisition des langues par des adultes migrants (Perdue 1993), l'extrait B du corpus d'une recherche sur la pédagogie des échanges (Saudan 2003). On y notera que dans chacune de ces interactions menée en français

\footnotetext{
${ }^{1}$ On préfère ici interaction à communication (voir Arditty dans ce numéro).
} 
(exolingue dans le premier cas, exolingue-bilingue dans le second), l'enquêteur, locuteur natif, sollicite et reçoit des informations et des explications sur un domaine connu de son interlocuteur, locuteur non natif.

A. “Comment c’est, un tambour ?“ (Giacomi, de Heredia 1986, 23)²

$1 \mathrm{E}:$ ah t'aimes pas / tu aimes pas le tambour là

2 IM : mais + pas tambour le / comment [sapil] ? /

$3 \mathrm{E}:$ / bandir c'est pas le tambour?

$4 \mathrm{E}:$ qu'est-ce que c'est + alors?

5 IM : pas [kone] bandir?

$6 \mathrm{E}$ : oui + tu appelles pas ça un tambour?

$7 \mathrm{IM}:$ comment?

$8 \mathrm{E}:$ c'est pas un tambour + pour toi ?

9 IM : non + tambour

$10 \mathrm{E}$ : comment c'est + un tambour?

$11 \mathrm{IM}$ : comment [se] un tambour ? + comme [li] bandir mais [sa] grand

$12 \mathrm{E}$ : ah d'accord + le bandir c'est petit

13 IM : oui le bandir [se] petit

B. "genau comme ça " (Saudan 1999, Annexe 8-29 K1, p. 9) 3

$\mathrm{G}$ : avec fraisage on fait les formes de la/

$\mathrm{E}$ : comment t'as fait pour les formes comment t'as $\mathrm{f} /$

$\mathrm{G}:$ aah ehm

$\mathrm{E}$ : tu pourrais vraiment me décrire tout ce que tu as fait en fait pour le faire

$\mathrm{G}$ : ehm je x commence avec le skizze avec des crayons nous ehm écrit des formes dans dans le boîte après

$\mathrm{E}$ : mais les formes tu les as fait pourquoi comment tu as est-ce que tu as pris le

l'instrument et tu as fait comm ça ou

$\mathrm{G}$ : oui oui

$\mathrm{E}:$ tu l'as mis $\underline{\text { sur le bois }}$ et puis t'as pris le crayon et t'as entouré

$\mathrm{G}: \quad \underline{\mathrm{mhm}} \underline{\mathrm{mhm}} \underline{\mathrm{mhm}}$

oui mais pas ehm x comme ça mais un peu mhm

$\mathrm{E}$ : un peu plus large

$G$ : mais pas also nicht nyd gnau

E : pourquoi pas

G : aah c'est pour fraisage c'est [rire] ça marche pas comme se fait genau comme ça

2 Il s'agit d'un extrait du corpus du programme ESF sur l'acquisition des langues par les adultes migrants (voir Perdue 1993). IM est un jeune arabophone arrivé récemment en France. E est l'enquêteur francophone natif. Le signe + indique une pause. Les crochets [] isolent une transcription phonétique. Noter que bandir a été transcrit en italique.

${ }^{3}$ Extrait du corpus de la thèse de V. Saudan (1999). Il s'agit ici d'un entretien entre un enquêteur francophone (E) et un jeune suisse germanophone (G), apprenti dans le domaine évoqué par le dialogue. 
Dans les deux cas (A et B), l'échange s'effectue dans la langue de celui qui est en position d'enquêteur, face à un «alloglotte» (locuteur non natif par rapport à la langue de l'échange), expert du domaine de savoir ou d'activité professionnelle faisant l'objet et la matière de l'échange. On trouvera dans ce numéro d'autres extraits (Yanaprasart, Champion) de corpus illustratifs d'une telle situation. Il en va différemment dans le cas de l'enquête linguistique classique de terrain lorsque le chercheur, même s'il est un linguiste expert, n'a qu'une maitrise partielle, voire rudimentaire, de la langue sur laquelle il enquête, et doit mener des interactions par divers moyens langagiers, le plus souvent autres que sa propre langue maternelle ou que d'autres idiomes éventuellement partagés avec ses informateurs.

1. Dans le cadre de la linguistique contemporaine, c'est chez Wolff $(1966 / 1959)^{4}$ qu'apparait l'une des premières mentions théorisées de la communication entre locuteurs de répertoires linguistiques différents. Dans son article intitulé Intelligibility and inter-ethnic attitudes, il pose explicitement la question du degré d'intelligibilité entre langues ou dialectes présumés plus ou moins proches, à travers l'intercompréhension entre leurs locuteurs respectifs, lorsqu'ils se trouvent en interaction. Cet auteur parle là, de façon récurrente, d'interlingual communication ${ }^{5}$, qu'il associe à la notion d'intelligibilité (intelligibility). Son questionnement, orienté vers l'identification et le classement typologique de langues et de dialectes (l'un des critères étant celui de l'intelligibilité entre ces idiomes, donc entre leurs locuteurs), est fondé là sur des enquêtes de terrain concernant des langues d'Afrique de l'Ouest (parlées au Nigéria, au Bénin et au Niger). Wolff constate d'abord des cas d'intercompréhension non réciproque ('non-reciprocal intelligibility') entre deux groupes linguistiques (ou dialectaux) malgré la proximité relative des idiomes; puis le fait que cette asymétrie constatée tient moins à des facteurs linguistiques qu’à des facteurs culturels, y compris à des rapports de domination ou de prédominance économique. Au-delà des contextes linguistiques,

plusieurs variables affectent et déterminent le degré de compréhension linguistique ou de communication interlinguistique.[...] L'existence d'une communication interlinguistique n'est pas forcément un indice d'une similarité entre deux [tels] dialectes. [...] L'intelligibilité, ou la communication interlinguistique, est fonction de tendances et de relations interculturelles. [...] Il est nécessaire de mener des recherches poussées sur la nature de l'intelligibilité et des conditions linguistiques non linguistiques de la communication interlinguistique. ${ }^{6}$ (pp. 442-443).

\footnotetext{
${ }^{4}$ La seconde date est celle de la parution originale. La première est celle signalée dans la bibliographie en fin d'article.

${ }^{5}$ Que l'on pourrait traduire, par exemple, par communication interlinguistique, communication interlinguale ou communication interlingue....

${ }^{6}$ Several variables affect and determine the degree of linguistic intelligibility or interlingual communication.[...] The existence of interlingual communication is not necessarily an indication of similarity between two such dialects [...] Intelligibility, or interlingual communication, is a function of intercultural trends and relationships [...] Intensive research into the nature of intelligibility and
} 
Rémy Porquier

Wolff met là en évidence, sans évoquer explicitement sa propre intervention de chercheur dans la quête ethnographique, un phénomène et une notion sans doute familières aux chercheurs de terrain en ethnographie et en linguistique : les enquêtes de terrain autres que celles menées par des locuteurs natifs de la langue à décrire impliquent de façon constitutive des interactions exolingues et/ou bilingues sous des formes diverses. Mais ces enquêtes de terrain ne représentent elles-mêmes qu'un cas spécifique, parmi bien d'autres, de telles interactions.

Bien que Wolff n'aborde pas là la dimension acquisitionnelle des interactions impliquées dans son travail d'enquête, celle-ci est nettement présente dans la recherche de terrain en ethnographie et en linguistique depuis - au moins - le début du $20^{\text {e }}$ siècle.

Ainsi, Boas écrit dès 1911 : " une certaine maîtrise de la langue est un moyen indispensable pour recueillir des informations précises et complètes, car le recueil d'information est effectué pour une large part en écoutant des conversations des autochtones et en participant à leur vie quotidienne, ce qui, pour l'observateur dépourvu d'une connaissance de la langue est complètement impossible » (1964-1911 : $16)^{7}$. Au chercheur de terrain se posent trois questions : apprendre pour parler? apprendre pour décrire? parler pour décrire ? Faut-il ou non avoir déjà des notions de la langue pour (mieux) la décrire ? Faut-il pouvoir, à quelque degré, converser soimême dans leur langue avec les informateurs? Sinon, comment recourir à des truchements, à des informateurs bilingues? Ces questions touchent déjà à la problématique plus large des interactions exolingues-bilingues (De Pietro 1988).

C'est ainsi qu'on trouvera, surtout à partir des années 1930, un ensemble de conseils, de directives ou de méthodes sur la gestion des échanges langagiers dans ce type d'enquête, voire des manuels ou des guides pour l'enquête de terrain et/ou pour l'apprentissage des langues locales par des intervenants de statuts divers.

Ainsi, Ida Ward (1939), linguiste africaniste, propose principes et conseils pratiques pour l'apprentissage d'une langue africaine sur le terrain, à l'intention de qui va travailler en Afrique comme commerçant, colon, missionnaire, anthropologue, éducateur ou administrateur (p. 5). Bloomfield publie en 1942 un Outline guide for the practical study of foreign languages, d'une quinzaine de pages, à destination d'apprenants adultes de langues non étudiées aux Etats-Unis d'alors dans les contextes scolaires, et donc entre autres aux enquêteurs de terrain. Nida (1950) publie un manuel d'apprentissage des langues à destination des missionnaires, dont certains sont euxmêmes ethnologues et/ou linguistes. Ces trois auteurs, alors linguistes de terrain chevronnés, associent dans leurs guides des notions et des méthodes de linguistique

into the linguistic as well as non-linguistic requisites of interlingual communication is needed. [notre traduction. Les termes soulignés en italique dans la traduction le sont par nous-même].

${ }^{7}$ « a command of the language is an indispensable means of obtaining accurate and thorough knowledge, because much information can be gained by listening to conversations of the natives and by taking part in their daily life, which, to the observer who has no command of the language, will remain entirely inaccessible ». [notre traduction] 
descriptive et des propositions pratiques et concrètes pour l'acquisition en contexte des langues visées 8 .

De tels manuels intègrent les apports progressifs de la linguistique structurale, de la méthodologie d'enquête de terrain et, plus tardivement, des outils technologiques d'enregistrement sonore?

On trouve plus tard, chez Samarin (Field linguistics, 1967), un véritable manuel de linguistique de terrain ${ }^{10}$, dans lequel ${ }^{11}$ il souligne divers aspects de la relation entre le recueil de données de terrain et l'apprentissage de la (des) langue(s), et que l'apprentissage de la langue peut être soit le but soit le moyen du recueil de données. Dans le premier cas, les données sont recueillies et traitées en fonction de besoins pratiques immédiats et personnels, et ce recueil est alors inadéquat ou insuffisant pour une visée descriptive, ne serait-ce qu'en raison du décalage temporel entre les deux entreprises, mais aussi du fait de la nature même des données. Mais Samarin expose en détail (p. 49-52) les raisons et l'intérêt, pour le chercheur, de conjuguer les deux visées :

a) l'apprentissage de la langue peut être à la fois une occupation et une distraction, l'acquisition d'un nouveau savoir(-faire) et un dérivatif (socialisant) dans l'isolement socioculturel du chercheur

b) une certaine connaissance de la langue locale facilite les contacts du chercheur avec la communauté hôte

c) une connaissance de la langue locale facilite le recueil d'un corpus plus étendu et plus diversifié.

d) une connaissance de la langue locale mène le linguiste à imaginer des constructions qui n'auraient pas été envisagées, et à une analyse plus explicite des problèmes rencontrés. Ses propres questionnements débordent alors, en le questionnant à son tour, le cadre du corpus qu'il a recueilli.

e) une connaissance de la langue locale permet au linguiste d'intégrer à son corpus externe un corpus "interne" qu'il peut exploiter pour son analyse de la langue. Il devient alors lui-même un informateur supplémentaire.

Comme on le voit, ces indications prennent en compte une entreprise très spécifique, dans laquelle les interactions exolingues, quelque forme qu'elles prennent (y compris lorsqu'elles associent ou mobilisent des interprètes ou truchements de circonstance), sont constitutives à la fois de l'objet et de la méthode d'investigation. Nous y reviendrons brièvement plus loin.

On notera seulement que si des linguistes ont proposé des manuels ou des guides pour l'apprentissage d'une langue ou de langues avant ou sur le terrain, nous

\footnotetext{
8 voir aussi, par exemple, Healey (1975) et Henry (1940).

${ }^{9}$ Ce n'est qu'à partir des années 1950 que le magnétophone a été utilisé pour des enquêtes linguistiques.

${ }^{10}$ Voir, pour le français, Thomas et al. 1971.

11 Samarin introduit un certain nombre de conseils pratiques et techniques pour l'enregistrement sonore sur magnétophone, pour le choix du matériel selon le contexte climatique et pour la conservation matérielle des enregistrements.
} 


\section{Rémy Porquier}

disposons par ailleurs de peu de rapports circonstanciés de tels linguistes sur leur propre apprentissage des langues sur lesquelles ils ont enquêté ${ }^{12}$.

A ce point, plusieurs observations sont utiles, en rapport avec les recherches alors à venir sur l'acquisition des langues, l'interlangue et les lectes d'apprenants et de locuteurs non natifs, la communication et l'interaction exolingues.

$1^{\circ}$ Les situations observées par Wolff illustrent un type de contact de langues. La notion de contact de langues, introduite et théorisée par Weinreich (" the languageusing individuals are [...] the locus of the contact », $1953: 1$ ) antérieurement à l'article de Wolff, aura également eu une influence décisive sur l'élaboration par Selinker (voir Selinker 1995, 26-40) de la notion d'interlanguage ${ }^{13}$.

$2^{\circ}$ Par ailleurs, les manuels d'enquête, comme les enquêtes elles-mêmes, introduisent très progressivement à partir des années 1930-1940 des techniques d'enregistrement sonore (disque puis magnétophones) qui permettent non seulement un meilleur recueil et un meilleur traitement des données orales, matériau ethnographique de première main, mais également la conservation des interactions orales entre chercheur, informateur(s) et éventuellement truchements bilingues. De tels documents constituent des échantillons spécifiques d'échanges exolinguesbilingues, dans lesquels la dimension métalinguistique et métacommunicative est forcément prégnante.

$3^{\circ}$ Le développement accéléré de l'enseignement des langues et de nouvelles méthodes d'enseignement, à partir des années 1950, suscite une diversité de recherches, tout d'abord en milieu guidé, et de publications ${ }^{14}$, qui s'orienteront ensuite progressivement, à partir des années 1970, vers l'acquisition et l'apprentissage des langues dans une diversité de contextes, par exemple chez des enfants et chez des adultes migrants, en milieux dits non guidés, et faisant alors une place primordiale aux compétences orales et aux interactions orales et à l'analyse de corpus oraux enregistrés.

$4^{\circ}$ Le développement parallèle des travaux en ethnographie de la communication suscite et explique des rapprochements ou des convergences avec la recherche en acquisition, quant aux contextes d'observation, au choix et au recueil de données, et aux méthodes d'analyse. La place faite aux interactions, et non plus seulement aux apprenants, dessine et sollicite à la fois d'autres champs d'observation et de recueil (voir 3 ci-dessous).

2. Le second repère, que nous situerons dans les années 1990, inclut également des développements très récents autour de l'an 2000. Il est caractérisé par la diversification des contextes d'investigation (voir Ellis 1987, Gajo et Mondada 2000,

\footnotetext{
12 On pourra cependant lire à ce propos les notations ironiquement désabusées de Barley (1992/1982, 68-69).

13 On notera également que la notion de mutual intelligibility évoquée par Wolff se retrouve en 1975, à propos d'interlanguage, chez Selinker, L., Swain, M., Dumas, G. (1975) : «The interlanguage hypothesis extended to children », Language Learning 25-1, 139-152.

14 On notera que la revue Language learning, la première spécialisée dans la piblication de travaux de recherche sur l'enseignement/apprentissage des langues, parait à partir de 1948. Pour un aperçu sur cette période, voir Stern 1983.
} 
Porquier et Py 2004) d'une part, des méthodes et procédures d'investigation d'autre part.

Au-delà des contextes, eux-mêmes très variés, d'apprentissage guidé, des recherches s'orientent progressivement vers l'acquisition en milieu naturel (ou «non guidé »), particulièrement chez des migrants adultes ${ }^{15}$. Chemin faisant, les travaux en viennent à identifier et à explorer des contextes, des situations et des interactions sollicitant un riche éventail de dispositifs, de principes et procédures de recueil de données et d'analyse (voir par exemple Perdue 1982, Larsen-Freeman et Long 1990, Seliger et Shohamy 1989).

Ces démarches se trouvent partiellement inspirées ou informées, au plan méthodologique, par des méthodes classiques d'enquête ethnolinguistique, par des tâches et activités usitées dans l'enseignement des langues, mais aussi par les apports de la psycholinguistique expérimentale, de la sociolinguistique, puis de l'ethnographie de la communication et de l'analyse conversationnelle. Elles intègrent en outre des méthodes de recueil de données, des "tâches » parfois originales, spécifiquement conçues ou adaptées selon les objectifs et les conditions de la recherche sur les interlangues et les interactions exolingues et bilingues.

$\mathrm{Si}$ les premiers travaux étaient plutôt centrés sur le recueil de corpus d'apprenants - de façon comparable à plusieurs égards aux travaux recueillant des corpus de locuteurs natifs de langues à décrire -, d'autres se sont progressivement centrés sur les interactions exolingues, en rapport ou non avec des trajectoires d'acquisition/apprentissage. D'où l'intérêt accru pour les interactions en milieu social et en milieu de travail intégrant diverses formes de communication exolingue et/ou bilingue $^{16}$.

Les travaux en France de l'équipe Langage et travail (voir notamment Borzeix et Fraenkel 2001) présentent une problématique de l'interaction langagière dans le monde et les activités du travail («la parole au travail ») ainsi que des propositions et des questionnements sur le recueil de corpus et la matière même des corpus dans divers contextes professionnels. Dans ce domaine de recherche, l'activité langagière est constitutive et en même temps constructrice de l'activité de travail, et de la coopération et de la coordination qui s'y gèrent et s'y négocient. L'action y est considérée comme une « construction située » (p. 24-25), inséparable de son contexte. Ce qu'illustrent entre autres les enquêtes sur l'information des voyageurs dans une gare (chap 8), ou sur la communication à l'hôpital (Chap. 13). Bien que ces travaux ne portent pas a priori sur la communication exolingue-bilingue, divers contextes professionnels et sociaux traités comportent une part de telles interactions.

Dans le cadre des interactions exolingues-bilingues, des recherches récentes (par exemple Cigada et al. 2001, Yanaprasart 2000, Kloster 2003) mettent en évidence la nature et des spécificités d'interactions en milieu professionnel (organismes internationaux, entreprises, restaurants, hôpitaux, sport professionnel, et même dans

\footnotetext{
15 Voir le projet ZISA dans Meisel, Clahsen et Pienemann, 1981, le projet ESF dans Perdue 1993, le projet de Pavie dans Giacalone Ramat, dir. 1995.

16 On distingue ici l'enseignement/apprentissage d'une langue à des fins professionnelles (comme le français sur objectifs spécifiques, ou English for specific purposes) des interactions effectives en milieu professionnel, même si celles-ci comportent naturellement souvent des dimensions acquisitionnelles.
} 
un Institut de langues). Bührig (2001), dans une enquête sur la communication en milieu hospitalier, étudie les interactions médecin-patient médiatisées (allemandportugais) par des truchements qui sont soit des membres bilingues du personnel hospitalier, soit des parents du patient.

L'enjeu est ici double : d'une part, identifier les espaces d'interaction accessibles où se donne cours la communication exolingue-bilingue sans contrôle externe d'un dispositif de recherche, d'autre part, disposer des moyens techniques de recueil in vivo de ces interactions ${ }^{17}$. L'enquêteur est là partiellement absent de cet espace ${ }^{18}$ en tant que partenaire de langage, ce qui redéfinit son rôle et son statut dans d'autres interactions. Le paradoxe de l'observateur n'est pas là évacué, mais seulement contenu ou déplacé.

D’autres travaux (Yanaprasart 2000 et dans ce numéro, Rosen 2001, Saudan 2003, Champion dans ce numéro) étudient les interactions exolingues dans le cadre de formations professionnelles incluant une formation en langue, ou de formations conjoignant les deux. L'apprentissage de la langue étant là, sous des formes diverses selon les contextes, couplé avec la formation professionnelle et le monde même du travail, les activités qui s'y donnent cours intègrent une pratique interactive et professionnelle de la langue, y compris dans des activités de simulation ou de jeux de rôle. Ici, la position du chercheur-enquêteur varie selon son rôle éventuel d'enseignant et/ou de formateur au métier proprement dit. Mais il peut s'agir de sa part, comme chez Rosen, Saudan et Champion, d'observation participante ; il peut en outre alors ajuster les activités en fonction d'objectifs de recherche préétablis.

Deux autres orientations récentes, d'inspiration explicitement ethnographique, sont illustrées par C. Roberts et al. 2001, et par Cambra Giné 2003.

Dans la première («Language learners as ethnographers »), les sujets apprenants sont directement impliqués dans un processus d'enquête, soit en les associant à sa démarche même, soit en les rendant eux-mêmes enquêteurs, de façon adaptée aux objectifs de leur formation. Ainsi, les séjours d'étude à l'étranger (qu'ils aient une visée linguistique, culturelle ou autre) peuvent être exploités pour des enquêtes de terrain, à caractère ethnographique et langagier (voir Roberts et al. 2001), avec pour finalité majeure le développement d'une compétence communicative interculturelle. Comme le soulignent les auteurs (p. 11), l'ethnographie est une méthode de recherche de terrain; dans le cas de l'expérience relatée, les apprenants - après quelque formation à cette démarche - conduisent eux-mêmes observation et recueil de données.

Pour Cambra Giné (2003), l'approche ethnographique de la classe de langue, qu'elle présente et relate (enseignement/apprentissage des langues en Catalogne), prend en compte le contexte et appréhende la classe de langue comme un lieu d'interaction sociale et de travail parmi d'autres, rejoignant ainsi les travaux menés

\footnotetext{
${ }^{17}$ Ainsi, une difficulté technique forte, mais surmontable, est d'enregistrer les interactions verbales entre médecin, infirmier et patient, ou entre des joueurs professionnels (de langues maternelles diverses) de football à l'entraînement et en match.

${ }^{18}$ Sauf à être lui-même un professionnel du domaine, associé ou délégué en la circonstance au dispositif de recherche.
} 
ailleurs sur la parole et les interactions au travail. Pour elle, cette démarche ${ }^{19}$ implique une approche participante: où l'observateur «se trouve dans le groupe avec la fonction d'observer, $[\ldots]$ son rôle est reconnu par les acteurs et [...] il est intégré dans le champ même de l'observation » (p. 17).

3. Cet élargissement de la recherche à des contextes de plus en plus diversifiés, à un large éventail de terrains, sollicite une variété de méthodes et de techniques d'enquête, et vient du même coup cristalliser des questions méthodologiques aigües, notamment quant à la nature des données et quant au statut et à la position du chercheur et à sa relation aux informateurs, ces derniers acteurs premiers des interactions qui font l'objet de l'investigation. Ces questions, qui ramènent à une problématique ancienne de la linguistique de terrain et de la recherche ethnographique, comme on l'a évoqué brièvement plus haut, se trouvent cependant posées là différemment, dans la perspective d'interactions situées entre acteurs sociaux.

La notion même de terrain se trouve là réexaminée : c'est au départ, dans la tradition de la linguistique dite de terrain, le terrain - le lieu et le matériau - du chercheur -, mais aussi celui de la langue et de la culture objets d'investigation, et donc le territoire de ses détenteurs. A propos des recherches sur l'acquisition des langues, Py (1993) identifie et caractérise le territoire de l'apprenant, sujet épistémique, comme lieu déterminé par sa position singulière vis-à-vis du système, de la norme et de la tâche.

Comme le montrent, de divers points de vue, Arditty et Vasseur (1999), Mondada (1998), Pallotti (2002) et Cambra Giné (2003), il s'agit en réalité d'un terrain d'interaction, que construisent, occupent et travaillent les participants, dans divers rôles respectifs. Qu'il s'agisse d'acquisition des langues ou d'interaction exolingue hors acquisition, on dépasse là une démarche (celle, initiale, de la linguistique de terrain, des recueils d'interlangues et de lectes d'apprenants) dans laquelle il y aurait un fournisseur de données et un solliciteur-collecteur de données. Ou bien le chercheur se trouve directement impliqué dans l'interaction selon un dispositif d'observation participante, voire comme acteur-observateur ; ou bien, observateur externe, il s'y trouve autrement impliqué comme récepteur, c'est-à-dire comme participant tiers, de façon simultanée lors de l'observation et du recueil, et éventuellement différée, lorsqu'il se fait transcripteur et analyste. Le double statut, fréquent, d'enquêteur-chercheur (celui qui recueille est aussi celui qui analyse) est aussi ancien que la linguistique de terrain. Plus récente est la prise en compte de son rôle et de sa place dans le dispositif d'enquête, les interactions objets d'enquête, et les interactions d'enquête elles-mêmes ${ }^{20}$. Mais la communication exolingue, si l'on revient aux premières élaborations de cette notion, apparait alors non seulement comme un objet d'investigation, mais comme constitutive de l'investigation. Les interactions exolingues de recherche constituent elles aussi un lieu spécifique d'activité sociale et professionnelle intéressant entre

\footnotetext{
${ }^{19}$ La démarche est dans cet ouvrage abondamment et soigneusement explicitée, y compris quant aux modalités de recueil et de transcription de données (Chapitres 3, 6 et Annexe).

${ }^{20}$ On renvoie ici aux échantillons présentés en introduction.
} 
Rémy Porquier

autres l'ethnographie de la communication et l'analyse conversationnelle, ainsi qu'un espace et un matériau de formation de chercheurs de terrain.

\section{BIBLIOGRAPHIE}

ARDITTY, J., VASSEUR, M.-TH. (1999) : «Interaction et langue étrangère : présentation ». Langages 134, 3-19.

BARLEY, N. (1982/1992) : The innocent anthropologist. London, British Museum Publications, 1982. [Trad. fr. par Marc Duchamp Un anthropologue en déroute. Paris, Payot, 1992].

BLOOMFIELD, L. (1942) : Outline guide for the practical study of foreign languages. Linguistic Society of America. Baltimore, Waverly Press.

BOAS, F. (1964/1911) : «Linguistic and ethnology », in HYMES (ed.) 1964, 15-26. [Repris de F. Boas, ed. (1911) : Handbook of American Indian Languages. Washington, Smithsonian Institution].

BORZEIX, A., FRAENKEL, B., (dirs.) (2001) : Langage et Travail. Communication, cognition, action. Paris, CNRS Editions.

BÜHRIG, K. (2001) «Interpreting in hospitals », in CICADA, S. \& al (2001), 108-119.

CAMPRA GINE, M. (2003) : Une approche ethnographique de la classe de langue. Paris, Hachette, coll. LAL.

CICADA, S., GILARDONI, S., MATTHEY, M., (dirs.) (2001): Comunicare in ambiente professionale plurilingue. Lugano, USI.

CORDER, S. P. (1967): “The significance of learners'errors”, International Review of Applied Linguistics (IRAL) 5 (4), 161-170. [Trad. fr. dans Langages 57, 1980, 9-15].

DE PIETRO, J. F. (1988) : "Vers une typologie des situations de contacts linguistiques», Langages et société 43, 65-89.

ELLIS, R. (ed.) 1987 : Second language acquisition in context. Englewood Cliffs, NJ, Prentice Hall International.

ELLIS, R. (1994) : The study of second language acquisition. Oxford, Oxford University Press.

ELLIS, R. \& ROBERTS, C. (1987) : «Two approaches for investigating second language acquisition in context », in ELLIS, R., (ed.) (1987), 3-29.

GAJO, L., MONDADA, L. (2000) : Interactions et acquisitions en contexte. Fribourg, Editions Universitaires Fribourg Suisse.

GAONAC'H, D. , PERDUE, C. (2000) : «L'acquisition d'une langue étrangère », dans KAIL, M. \& FAYOL, M. L'acquisition du langage. Vol. II, Paris, PUF, 215-246. 
GIACALONE RAMAT, A., (dir.) (1995) : L'acquisition de l'italien langue étrangère. Acquisition et Interaction en Langue Etrangère 5.

GIACOMI, A., DE HEREDIA, C. (1986): «Réussites et échecs dans la communication linguistique entre locuteurs francophones et locuteurs immigrés ». Langages 84, 9-24.

HEALEY, A., (ed.) (1975) : Language learner's field guide. Ukarumpa (New-Guinea), Summer Institute of Linguistics.

HENRY, J. (1940): “A method for learning to talk primitive languages". American Anthropologist 42, 635-641.

HYMES, D., (ed.) 1964 : Language in culture and society. New-York, Harper \& Row.

KLEIN, W. (1991) : "SLA theory: prolegomena to a theory of language acquisition and implications for theoretical linguistics », in T. HUEBNER \& C.A. FERGUSON (eds.) : Crosscurrents in second language acquisition and linguistic theories. Amsterdam, John Benjamins, 169-194.

KLOSTER, N. (2003) : La communication exolingue chez des joueurs de football professionnels brésiliens en France. Mémoire de DEA en Sciences du langage, Université Paris X.

LARSEN-FREEMAN, D., LONG, M.H. (1990): An introduction to second language acquisition research. London \& New-York, Longman.

MEISEL, J., CLAHSEN, H. \& PIENEMANN, M. (1981) : «On determining developmental stages in natural second language acquisition », Studies in second language acquisition 3 (2), 109-135.

MONDADA, L. (1998): "Technologies et interaction dans la fabrication du terrain du linguiste ", dans MAHMOUDIAN, M. \& MONDADA, L. (dirs.) : Questionner les pratiques, les méthodes, les techniques de l'enquête. Lausanne, Cabiers de l'ISL 10, 3968.

NIDA, E. (1947) : «Field techniques in descriptive linguistics », International Journal of American Linguistics, 13, 138-146.

NIDA, E. 1950 : Learning a foreign language : a handbook for missionaries. New-York, Foreign missions conference of North America.

NOYAU, C., PORQUIER, R., (dirs.) (1985) : Communiquer dans la langue de l'autre. Saint-Denis, Presses Universitaires de Vincennes.

PALLOTTI, G. (2002) : «La classe dans une perspective écologique de l'acquisition», Acquisition et Interaction en Langue Étrangère 16, 165-197.

PERDUE C., (ed.) (1982) : Second language acquisition by adult immigrants. A field manual. Strasbourg, European Science Foundation. [rééd. 1984, Rowley, Newbury House].

PERDUE, C., (ed.) (1993) : Adult second language acquisition : cross linguistic perspectives. Vol. II : The results. Cambridge, Cambridge University Press.

PORQUIER, R., PY, B. (2004) : Apprentissage d'une langue étrangère : contextes et discours. Paris, Didier, coll. Essais. 
Rémy Porquier

PUJOL, M. \& VERONIQUE, D. (1991): L'acquisition d'une langue étrangère: recherches et perspectives. Cabiers des Sciences de l'Education 63, Université de Genève.

PY, B. (1993) : "L'apprenant et son territoire: système, norme et tâche ", Acquisition et Interaction en Langue Etrangère 2, 10-24.

ROBERTS, C., BYRAM, M., BARO, A., JORDAN, S., \& STREET, B. (2001) : Language learners as ethnographers. Clevedon, Multilingual matters.

ROBERTS, C. \& SIMONOT, M. (1987): “This is my life : how language acquisition is interactionally accomplished ", in ELLIS, R., (ed.) (1987), 133-148.

ROSEN, E. (2001): Apprendre une langue en communiquant. Interlangue et communication exolingue/endolingue en contexte. Thèse de doctorat en Sciences du langage, Université Paris-X Nanterre.

SAMARIN, W. J. (1967) : Field linguistics. New-York, Holt, Rinehart \& Winston.

SAUDAN, V. (1999) : Approche communicative et pédagogie des échanges. Thèse, Université de Bâle.

SAUDAN, V. (2003) : Approche communicative et pédagogie des échanges. Apprendre une langue seconde à l'intérieur et à l'extérieur de l'école. Bâle, Acta Romanica Basiliensia.

SELIGER, H. W., SHOHAMY, E. (1989) : Second language research methods. Oxford, Oxford University Press.

SELINKER, H. W. (1972) : «Interlanguage », International review of Applied Linguistics 10, 219231.

SELINKER, L. (1995) : Rediscovering interlanguage. London \& New York, Longman.

STERN, H. H. (1983) : Fundamental concepts of language teaching. Oxford, Oxford University Press.

THOMAS, J., HAUDRICOURT, A.G., CAPRILE, J.P. (1971) : Enquête et description des langues à tradition orale. Paris, SELAF.

VERONIQUE, D. (1992) : «Recherche sur l'acquisition des langues secondes : un état des lieux et quelques perspectives ", Acquisition et Interaction en Langue Etrangère 1, 5-35.

VERONIQUE, D. (1997) : «La didactique des langues et des cultures face à l'apprenant de langues étrangères et à ses activités d'appropriation », Etudes de linguistique appliquée 105, 93-110.

VOGEL, K. (1995) : L'interlangue, la langue de l'apprenant. Toulouse, Presses universitaires du Mirail.

WOLFF, H. (1964/1959) : «Intelligibility and inter-ethnic attitudes », in HYMES, D. (ed.) 1964 [Version initiale : Anthropological linguistics, 1959, 1-3].

YANAPRASART, P. (2000) : Langue et culture dans l'enseignement du français en Thaïlande. Thèse de doctorat, Université de Neuchâtel. 